\title{
La conclusion de la Critique de la raison pure
}

\author{
par Jean Grondin, Québec
}

Il est bien connu que le problème fondamental de la Critique de la raison pure est celui de la possibilité de la métaphysique comme science. C'est le problème que pose la question directrice de l'ouvrage: comment des jugements synthétiques a priori sont-ils possibles? Ce qui est beaucoup moins connu et infiniment moins évident, c'est la réponse que Kant a donnée à ce problème capital, dont il a par ailleurs si bien marqué l'urgence. Or comment la métaphysique est-elle possible selon Kant? Où Kant répondil de manière claire et distincte à la question du bien-fondé des jugements synthétiques $a$ priori en métaphysique?

L'incertitude qui plane sur cette réponse n'est pas peu responsable de la diversité des interprétations qu'on a pu offrir de la solution kantienne au dilemme de la métaphysique. Pour les uns, Kant est souvent apparu comme celui qui aurait tout simplement voulu liquider la métaphysique en lui lançant le défi de rendre compte de la légitimité de ses propres jugements, lecture iconoclaste qui s'est maintenue depuis le mot de Mendelssohn sur l'alles zermalmenden Kant jusqu'à l'interprétation récente d'Arsenij Gulyga $^{1}$. Pour d'autres, la métaphysique serait devenue chez Kant une théorie des sciences exactes, des principes qui sont à la base du savoir scientifique ou, plus généralement, de notre perception du réel. On pourrait parler ici d'une «métaphysique de l'expérience», selon l'expression quasi paradoxale de H.J. Paton ${ }^{2}$. D'autres, prenant à la lettre le texte célèbre où Kant dit avoir dû supprimer le savoir pour faire place à la foi, voient dans la philosophie morale, que l'échec de la métaphysique théorique propulserait au rang de prima philosophia, l'issue véritable de la métaphysique kantienne. D'autres, enfin, les partisans de ce que l'on a appelé la lecture métaphysique de Kant, ont cru que l'auteur de la Critique avait voulu sauver et conserver l'essentiel de la métaphysique classique, aristotélico-leibnizienne, celle qui aspire à un savoir qui dépasse le cadre restreint de l'expérience.

Si autant d'interprétations ont pu être avancées, c'est que la Critique de la raison pure ne paraît jamais répondre de manière concluante à la question de la possibilité de la

1 M. Mendelssohn, Schriften zur Philosophie, Ästhetik und Apologetik, Georg Olms, Hildesheim, 1968, t. I, 299. A. Gulyga, Immanuel Kant, Suhrkamp, Frankfurt am Main, 1985, 152: „Kant hat auf die Frage zu Anfang der Kritik ,wie ist Metaphysik als Wissenschaft möglich?' faktisch keine Antwort gegeben. Durch seine transzendentale Dialektik hat er alle dogmatischen Gebäude in dieser Sphäre zerstört."

2 H.J. Paton, Kant's Metaphysics of Experience, Allen and Unwin, London, 1936, 4th edition: 1965. 
métaphysique et de ses jugements synthétiques a priori. Il ne semble pas y avoir de texte dans la première Critique qui affirme en toutes lettres: voici comment se résout le problème de la métaphysique, voici comment des jugements synthétiques de la raison pure sont possibles. On ne trouve pas, autrement dit, de conclusion à la Critique de la raison pure ou, ce qui est plus habituel chez Kant, de «remarque finale» où le philosophe ferait un bilan de ses recherches et condenserait sa réponse à la question inaugurale de son œuvre, magistralement posée dans l'Introduction.

Et pourtant, une telle réponse, une telle «conclusion», doit bien se trouver quelque part dans la Critique de la raison pure, car c'est très précisément pour résoudre ce problème de la possibilité de la métaphysique que la Critique a été instituée. Conçue comme propédeutique à la métaphysique, la Critique a pour fonction expresse de sonder la possibilité du jugement synthétique a priori. Sa raison d'être consiste à établir la crédibilité d'une métaphysique rationnelle. «Cette recherche, que nous ne pouvons pas nommer proprement doctrine, mais seulement critique transcendantale, parce qu'elle n'a pas pour visée l'extension des connaissances elles-mêmes, mais seulement leur rectification (Berichtigung), et qu'elle donne la pierre de touche de la valeur ou du défaut de valeur de toutes les connaissances a priori, est ce dont nous nous occupons maintenant. Une telle critique est par suite une préparation, autant que possible, pour un organon, et si celui-ci devait ne pas réussir, du moins pour un canon de ces connaissances a priori, d'après lequel en tout cas le système complet de la philosophie de la raison pure ... pourrait être un jour présenté.» ${ }^{3}$ Le système complet de la raison pure, que Kant annonce dans ce contexte sous le titre de «philosophie transcendantale», c'est donc ce qui doit venir après la critique transcendantale ou la critique de la raison pure, la propédeutique qui est censée fournir la pierre de touche du système de la métaphysique. «Un système de tels concepts s'appellerait», promet Kant au conditionnel, "philosophie transcendantale. Mais cette philosophie est encore trop à son tour pour commencer» ${ }^{4}$. Trop parce qu'on ne sait pas encore si et comment une telle connaissance métaphysique est possible. D'où la nécessité d'une propédeutique à la philosophie transcendantale, c'est-à-dire d'une «critique transcendantale» qui doit déterminer la pierre de touche de toutes les connaissances a priori. La critique transcendantale, qu'est la première Critique, veut justifier la possibilité d'une philosophie trancendantale ou d'une métaphysique, encore à déployer. Aujourd'hui, on entend souvent par philosophie «transcendantale» une réflexion qui porte sur les «conditions de possibilité» de quelque chose. Or, chez Kant, ce type de réflexion incombe à la critique transcendantale. Pour une philosophie transcendantale, telle que l'entend Kant, la question des «conditions de possibilité» est déjà, en principe, résolue.

${ }^{3}$ B 26; Critique de la raison pure, trad. par A.J.L. Delamarre et F. Marty, in E. Kant, CEuvres philosophiques, t. I, Gallimard, Bibliothèque de la Pléiade, Paris, 1980, 777. Cette traduction est celle que nous citerons dans cet article. Par commodité, on indiquera aussi la référence à la traduction de A. Tremesaygues et B. Pacaud (sigle: TP), P.U.F., Paris, 1944, 7e édition, 1971. Pour le texte ici cité de B 26, cf. TP 46.

${ }^{4}$ B 25; Euvres, 777; TP 46. 
La critique transcendantale de 1781 veut ainsi fixer l'usage métaphysique légitime de la raison pure afin de rendre possible le déploiement d'une métaphysique. La préface à la seconde édition définira donc cette critique comme «traité de la méthode» (Traktat von der Methode), lequel, précise Kant, n'est pas encore «un système de la science même» ${ }^{5}$ - ce que sera la métaphysique ou la philosophie transcendantale. La Critique se présente de la sorte comme la tentative (Versuch) «de changer la démarche (Verfabren) jusqu'ici suivie en métaphysique, opérant ainsi en elle une complète révolution» ${ }^{6}$.

En lui-même, le projet d'une critique transcendantale de la raison pure, qui s'institue en prolégomènes de toute métaphysique future, est d'une remarquable transparence. Ce qui est moins clair, c'est sa réalisation, sa solution, sa conclusion, en un mot, le sens exact de la transformation qu'il s'agit d'introduire dans la méthode jusqu'à maintenant préconisée en métaphysique. En quoi consiste cette nouvelle méthode de la raison pure? À la recherche de cette conclusion de la Critique, on partira, comme il se doit, de la formulation initiale du problème de Kant: comment des jugements synthétiques a priori sont-ils possibles?

On sait que pour la Critique de la raison pure, tout comme les Prolégomènes de 1783, ce problème se subdivise en trois sous-questions: comment des jugements synthétiques a priori sont-ils possibles 1 . en mathématiques pures, 2 . en physique pure et 3 . en métaphysique? Triple question qui se laisse aussi poser de la manière suivante: comment la mathématique pure est-elle possible? comment la physique pure est-elle possible? comment la métaphysique est-elle possible comme science? Dans la présentation des Prolégomènes, qui correspond sur ce point assez bien à l'architecture de la Critique, la première question relève de l'Esthétique transcendantale, la seconde de l'Analytique et la troisième de la Dialectique. De fait, l'Esthétique rend compte de la possibilité des jugements synthétiques en mathématiques en invoquant comme troisième terme l'intuition pure de l'espace et du temps, au sein de laquelle peuvent être construites et vérifiées, parce que susceptibles d'illustration, les connaissances synthétiques a priori de la mathématique pure. Au fil d'une analyse encore plus complexe, que nous ne reproduirons pas ici', l'Analytique tente, de son côté, de justifier les propositions synthétiques a priori de la physique pure, consignées dans le système des principes de l'entendement pur, en s'appuyant sur le troisième terme que sera l'«expérience possible». Quant à la Dialectique, elle est bel et bien consacrée aux prétentions proprement métaphysiques de la raison pure. Seulement, le cas de la Dialectique est un peu particulier du fait que Kant n'y aboutit pas, comme dans l'Esthétique et l'Analytique, à un résultat vraiment positif, entendons à une solution du problème de la possibilité de la métaphysique comme science. Kant l'avoue lui-même, et de plusieurs façons. Tout d'abord lorsqu'il divise sa Logique transcendantale en Analytique et en Dialectique, il spécifie bien que la première révèle la logique du vrai et la seconde la

5 B XXII; Euvres, 743; TP 21.

6 Ibid.

7 Cf. à ce sujet le chapitre III de notre étude Kant et le problème de la philosophie: l'a priori, Vrin, Paris, 1989. 
logique de l'illusion ${ }^{8}$. Est-ce à dire que la métaphysique se trouve condamnée à la fausseté? Le domaine de l'a priori susceptible d'être connu doit-il se limiter au champ de la mathématique et de la physique? Ensuite, il reconnaît souvent que la tâche de la Dialectique demeure, quant à l'essentiel, purement négative. N'a-t-elle pas pour objet propre le règne de l'illusion? «La seconde partie de la logique transcendantale doit donc être une critique de cette apparence dialectique, non comme un art de susciter dogmatiquement cette apparence ..., mais comme une critique de l'entendement et de la raison à l'égard de leur usage supraphysique, pour mettre à découvert l'apparence fausse où conduisent des ambitions sans fondement.»9

Faut-il en conclure à l'impossibilité de la métaphysique? Ce serait sans doute la conclusion à tirer si la Dialectique était le dernier mot et la dernière section de la Critique de la raison pure. Or après la Dialectique vient une «Méthodologie» (Metbodenlebre). Nous aimerions montrer que cette théorie de la méthode, loin d'être un simple appendice à la Critique, comme on le pense souvent, représente le véritable aboutissement de l'entreprise critique, c'est-à-dire le versant positif de la Dialectique, le lieu où s'esquisse une solution au problème de la métaphysique dans le projet critique de Kant. Sans cette issue positive, la question de la possibilité des jugements synthétiques a priori reste sans réponse.

Rappelons en premier lieu que la Méthodologie représente la seconde grande partie de toute la Critique, après la «Théorie transcendantale des éléments» (qui comprend l'Esthétique et la Logique, analytique et dialectique). Ce n'est pas rien. La division première d'une critique transcendantale est donc celle d'une théorie des éléments et d'une théorie de la méthode ${ }^{10}$. Si à la méthodologie revient l'honneur d'être la dernière partie de l'ouvrage, c'est que l'idée même d'une méthodologie est nécessairement liée au projet initial d'une critique de la raison pure. La Critique se définit, on s'en souvient, comme «traité de la méthode» qui veut opérer une révolution fondamentale en métaphysique en mettant à découvert la pierre de touche des connaissances métaphysiques. La Critique de la raison pure, en son projet, n'est rien d'autre qu'une tentative de révolutionner la métaphysique en proposant une nouvelle méthodologie de la raison pure qui pourra servir de fondement à l'édification d'une métaphysique véritable. Il y a donc lieu de réapprendre à voir dans la méthodologie l'aboutissement logique de l'investigation critique de Kant. Dans cette perspective, on pourrait presque dire que tout le propos de la «théorie transcendantale des éléments» n'aura été que de débroussailler les éléments (intuitions, concepts, idées) de la conaissance pure en vue de l'institution d'une méthodologie de la raison pure, instaurée en tant que propédeutique à la philosophie transcendantale, qui reste à élaborer pour elle-même. En exagérant peut-être, on serait ainsi portés à croire que toute la critique de la raison pure se cache

8 Nous reprenons ici la traduction de Schein par «illusion» proposée par R. Theis, De l'Illusion transcendantale, in Kant-Studien, 76, 1985, 120-137, mais conserverons la traduction par «apparence» dans les citations tirées de l'édition de la Pléiade.

9 A 63-4/B 88; Euvres, 821-2; TP 83-4.

10 Cf. A 15/B 29; CEuvres, 779-80; TP 48-9. 
finalement dans la Méthodologie. Chose certaine, l'instauration d'une métaphysique nouvelle n'a pas eu lieu dans la théorie transcendantale des éléments.

Le but d'une critique de la raison pure est d'évaluer la possibilité de la métaphysique, d'une connaissance synthétique a priori ou par raison pure. La critique transcendantale ainsi constituée se recommande comme propédeutique indispensable à la crédibilité d'une science métaphysique. Avant de construire un système de métaphysique, tâchons, exhorte Kant, de réfléchir une fois pour toutes sur les fondements du savoir a priori que veut être la métaphysique. Ce type de réflexion préalable sur les fondements de la science relève en propre de ce qu'on appelle la «méthode» (on pensera évidemment au «Discours de la méthode» de Descartes). La méthodologie sera ainsi la théorie de la méthode qu'il convient d'imposer à la raison pure si elle doit parvenir à une connaissance a priori et métaphysique qui soit fondée. La méthodologie critique contiendra donc les prolégomènes à l'usage justifié de la raison pure en métaphysique. Par «théorie transcendentale de la méthode», on entendra par suite, selon la définition expresse de Kant, «la détermination des conditions formelles d'un système complet de la raison pure ${ }^{11}$, encore à développer sous le titre de métaphysique. Il y va de l'élucidation des conditions de l'usage légitime de la raison pure en vue d'une métaphysique à venir. La Critique de la raison pure n'a pas de tâche plus essentielle.

Tout de suite après en avoir défini le projet, qui va donc de soi après la théorie transcendantale des éléments, Kant expose le plan de sa méthodologie: «À cette fin [c'est-à-dire: la détermination des conditions formelles d'un système complet de la raison pure)] nous aurons à nous occuper d'une discipline, d'une canon d'une architectonique, et enfin d'une histoire de la raison pure» ${ }^{12}$. Après avoir parcouru la Dialectique, on comprend aisément que la Méthodologie doive s'ouvrir sur une «discipline» de la raison pure. La raison métaphysique ayant naturellement tendance à succomber à l'illusion transcendantale, la raison pure soucieuse de sa propre méthode a en effet d'abord besoin d'une discipline, c'est-à-dire d'une «instruction à caractère négatif», d'après l'expression de Kant ${ }^{13}$. Sur ce point, la Discipline de la raison pure redouble l'effort thérapeutique et critique, au sens négatif du terme, de la Dialectique. Ce n'est pas cette discipline qui répondra de façon positive à la question de la possibilité de la métaphysique.

Ce n'est que dans son deuxième volet, le Canon de la raison pure, que la Méthodologie dégagera le nouveau fondement d'une métaphysique. L'idée d'un «canon» de la raison pure avait du reste déjà été évoquée dans l'Introduction de l'ouvrage, dans l'un de ses passages les plus stratégiques, là où s'était défini le projet de la Critique. Recitons l'essentiel du texte de Kant: «Une telle critique est par suite une préparation ... pour un canon de ces connaissances a priori, d'après lequel ... le système complet de la philosophie de la raison pure ... pourrait être un jour présenté» ${ }^{14}$. Où trouver ce

11 A 707-8/B 735-6; Euvres, 1294; TP 489.

12 A 708/B 736; Euvres, 1294; TP 489.

13 A 709/B 737; CEuvres, 1295; TP 491.

14 B 26; Euvres, 777; TP 46. 
canon de l'usage légitime de la raison pure, but de la première Critique, sinon dans son «Canon de la raison pure»?

Kant fournit une définition générale, mais claire, de ce qu'il faut comprendre par canon: «j'entends par canon l'ensemble des principes a priori du légitime usage de certains pouvoirs de connaître en général» ${ }^{15}$. Si ce pouvoir de connaître est la raison pure, son Canon indiquera nécessairement «l'ensemble des principes a priori du légitime usage de la raison pure». Cette définition associe on ne peut plus intimement le Canon de la raison pure au projet rigoureux d'une critique de la raison pure. Définir les principes a priori de l'usage légitime de la raison pure, n'est-ce pas l'objectif le plus évident de la Critique ${ }^{16}$ ?

Mais la Dialectique n'a-t-elle pas montré qu'il était vain d'espérer quelque connaissance métaphysique par raison pure? N'a-t-elle pas consigné dans toutes ses possibilités les raisonnements sophistiques de la raison pure «dans les archives de la raison humaine afin que l'on puisse éviter à l'avenir de semblables errements» ${ }^{17}$ ? Cependant, cet échec dialectique ne concerne que la raison théorique ou spéculative, celle qui s'appuie uniquement sur les raisonnements de la raison pure pour arriver à des connaissances $a$ priori. Cet échec de la raison pure théorique reste inexorable et Kant prend la peine de souligner qu'il ne peut $\mathrm{y}$ avoir de «canon» de cette raison pure, mais seulement une discipline négative ${ }^{18}$. Si canon de la raison pure il y a, il ne concernera que l'usage pratique de la raison pure, que Kant présente comme une issue tout à fait nouvelle, qu'il s'agit maintenant d'explorer: «Par conséquent, s'il y a quelque part un usage légitime de la raison pure, auquel cas il doit y avoir aussi un canon de cette raison pure, alors ce canon ne concernera pas l'usage spéculatif mais l'usage pratique de la raison. C'est donc cet usage que nous allons maintenant rechercher» ${ }^{19}$. Kant précise lumineusement que s'il y a usage légitime de la raison pure, il doit y en avoir un canon. La détermination de l'usage justifié de la raison pure, tâche première de la Critique, passe immanquablement par un «canon» de cette raison.

Kant souligne aussitôt l'urgence de son propos en intitulant la première section $\mathrm{du}$ Canon, qui s'ouvre après le texte que nous venons de citer, «De la fin dernière de

15 A 796/B 824; Euvres, 1359; TP 538.

${ }^{16}$ Cf. H. Heimsoeth, Transzendentale Dialektik, Band IV: Die Methodenlehre, Walter de Gruyter, Berlin/New York, 1971, 743: „Daß das gesamte Unternehmen unseres Werkes: Kritik als ,Propädeutik' und ,Traktat von der Methode' der Metaphysik, in Umänderung bisherigen Verfahrens, schließlich auf einen ,Kanon' abzielt, d.h. auf Grundsätze des ,richtigen', jeder Kritik standhaltenden Vernunftgebrauches - das ist schon in der Einleitung vorausfixiert."

17 A 704 /. B 732; CEuvres, 1291; TP 485.

${ }_{18}$ Cf. A 796/B 825; CEuvres, 1359; TP 539; «Or, suivant toutes les preuves qui ont été données jusqu'ici, toute connaissance synthétique de la raison pure dans son usage spéculatif est totalement impossible. Il n'y a donc pas de canon de l'usage spéculatif de la raison (car cet usage est dialectique de part en part), mais toute logique transcendantale n'est à cet égard que discipline.»

19 A 796-7/B 824-5; CEuvres, 1359; TP 539. C'est nous qui soulignons «doit». 
l'usage pur de notre raison». À n'en pas douter, on touche aussi au but final, au point d'arrivée de l'ensemble de la Critique de la raison pure, qui suspendra, comme on le verra, la possibilité de la métaphysique à l'intérêt pratique de notre raison pure. L'analyse de Kant commence par reprendre les trois objets de l'usage de la raison, tel qu'élaboré dans la Dialectique: la liberté de la volonté, l'immortalité de l'âme et l'existence de $\mathrm{Dieu}^{20}$. Autant d'objets qui nous intéressent au plus haut point, mais l'intérêt que nous leur portons n'est pas principalement d'ordre théorique selon Kant ${ }^{21}$. Par là, Kant veut simplement dire que ces objets, pourraient-ils être connus, ne nous seraient d'aucune utilité «in concreto, c'est-à-dire dans l'étude de la nature ${ }^{22}$. L'intérêt que nous portons à ces idées, prétend Kant, est proprement pratique. «Est pratique tout ce qui est possible par liberté» ${ }^{23}$. Or, argumente essentiellement Kant, les conditions de l'exercice de notre liberté ne sont pas seulement empiriques (sinon la morale se réduirait à une anthropologie pragmatique). Il est aussi des lois morales qui relèvent en propre de notre raison et qui commandent de manière inconditionnée et universelle. Il y a donc un «efficace» de la raison pure qui n'est pas de nature théorique et dont un canon sera possible. «Des lois pures pratiques au contraire, dont le but serait donné tout à fait a priori par la raison et qui ne commanderaient pas d'une manière empiriquement conditionnée mais absolue, seraient des produits de la raison pure. Or, telles sont les lois morales, et par conséquent seules elles appartiennent à l'usage pratique de la raison pure et permettent un canon ${ }^{24}$."

L'intérêt «pratique» que nous vouons aux trois idées de la raison, vers lesquelles sont dirigés «tous les préparatifs de la raison dans le travail que l'on peut appeler philosophie pure», a comme fin de savoir «ce qu'il faut faire, si la volonté est libre, s'il y a un Dieu et une vie future» ${ }^{25}$. Prétendant ici que la liberté pratique peut être connue par expérience, thèse qui na sera pas d'une constance absolue chez Kant, et qu'elle relève de ce fait de la philosophie spéculative, Kant limitera, dès la première Critique, le propos du canon de la raison pure pratique à deux objets: «par conséquent, dans un canon de la raison pure, nous n'avons à nous occuper que de deux questions qui concernent l'intérêt pratique de la raison pure, et relativement auxquelles un canon de son usage doit être possible, à savoir: Y a-t-il un Dieu? Y a-t-il une vie future?» ${ }^{26}$. L'objet pratique, voire existentiel, du canon est bien circonscrit.

Il rejoint aussi la préoccupation majeure de Kant dans son interrogation sur la possibilité de la métaphysique. La métaphysique dont la Critique veut sonder la viabilité ne vise pas avant tout l'érection d'un système scolastique qui offrirait des

20 A 798/B 826; Euvres, 1360; TP 539.

21 A 798/B 826; CEuvres, 1360; TP 539: «A l'égard de tous les trois l'intérêt purement spéculatif de la raison n'est que très faible».

22 A $798 /$ B 826; Euvres, 1360; TP 539.

23 A 800/B 828; Euvres, 1361; TP 540.

${ }_{24}$ A $800 /$ B 828; Euvres, 1362; TP 541.

25 Ibid.

26 A 803/B 831; CEuvres, 1364; TP 542. 
définitions a priori de tous les concepts en déclinant une characteristica universalis. La métaphysique kantienne est d'emblée intéressée. Elle veut répondre aux inquiétudes proprement métaphysiques de l'homme, celles qui constituent ce que Kant nomme la metaphysica naturalis, qui a toujours existé et qui existera toujours à titre de disposition naturelle ${ }^{27}$. Ses problèmes essentiels sont ceux de l'existence de Dieu et de l'immortalité de l'âme. Or cette métaphysique peut-elle atteindre le statut de science? Peut-il y avoir une connaissance scientifique qui réponde aux aspirations métaphysiques les plus élémentaires de l'homme, lequel ne peut «jamais se satisfaire avec ce qui est temporel ${ }^{28}$ ? Cette soif d'absolu se condense dans la double question: y a-t-il un Dieu? y a t-il une vie future?

Même si ces questions concernent au plus près l'usage dit pratique de la raison, il ne faut cependant jamais perdre de vue l'ambition théorique de l'examen de la raison pratique dans la première Critique. Il s'agit toujours de découvrir la pierre de touche d'une métaphysique, fil conducteur qui aiguille encore la Canon de la raison pure. Après avoir une nouvelle fois rappelé que la raison pure théorique succombe à une dialectique naturelle et qu'une discipline doit censurer, Kant note au début de son Canon: «Cependant (indessen) il doit y avoir quelque part une source de connaissances positives (einen Quell von positiven Erkenntnissen) qui appartiennent au domaine de la raison pure, et qui ne sont peut-être une occasion d'erreur que par l'effet d'un malentendu, mais qui en réalité constituent le but du zèle de la raison ${ }^{29}$. Bien que seul l'usage pratique de la raison pure soit à l'étude, on n'y trouvera pas moins la source (Quell) ou le fondement de connaissances positives par raison pure, donc métaphysiques. C'est ce fondement qu'il appartient en propre à la Critique de la raison pure de dégager en toute clarté afin de pouvoir édifier une métaphysique. La voie qui mène à une connaissance métaphysique réelle, synthétique a priori et légitime, passe par l'étude des principes de la raison pure pratique. C'est le sens de la révolution méthodologique que Kant tente d'introduire dans la méthode suivie jusqu'à maintenant en métaphysique. L'enjeu n'est pas mince.

$\mathrm{La}$ «fin dernière de l'usage pur de notre raison» (première section) étant fixée - elle tient dans la question: $\mathrm{y}$ a-t-il un Dieu et une vie future? -, c'est à la deuxième section du Canon qu'il reviendra de définir plus précisément cette voie, la seule qui reste ouverte à la métaphysique. Elle s'intitule «De l'idéal du souverain bien comme fondement pour la détermination de la fin dernière de la raison pure». Son premier paragraphe résume le chemin parcouru par la raison à travers la Dialectique transcendantale: la raison nous a menés vers des idées spéculatives, mais «celles-ci, à leur tour, ont fini par nous ramener à l'expérience et ont ainsi rempli son dessein d'une manière utile, il est vrai, mais nullement conforme à notre attente» ${ }^{30}$. En d'autres mots, le résultat de la Dialectique est fort honorable, mais il ne répond pas à nos attentes

27 B 21; CEuvres, 774; TP 44.

28 B XXXIII; Cuvres, 749; TP 25.

29 A 795-6/B 823-4; CEuvres, 1358; TP 538.

30 A 804/ B 832; Euvres, 1364-5; TP 543. 
puisqu'il laisse entière la question de la possibilité de la métaphysique, n'apportant notamment aucune réponse probante aux questions les plus vitales, celles de l'existence de Dieu et de l'immortalité. La perspective d'une assise pratique à la métaphysique s'ouvrira à cet endroit bien précis: «Or, il nous reste encore un essai à faire, c'est de chercher s'il y a aussi une raison pure dans l'usage pratique, si dans cet usage elle nous conduit à des idées qui atteignent les fins suprêmes de la raison pure que nous avons indiquées ..., et si par conséquent elle ne pourrait pas nous accorder au point de vue de son intérêt pratique ce qu'elle nous refuse absolument au point de vue de l'intérêt spéculatif ${ }^{31}$. Évoquant à une étape aussi décisive de sa réflexion l'intérêt de la raison, Kant présente alors les trois célèbres questions où se concentre tout intérêt de ma raison: que puis-je savoir? que dois-je faire? que m'est-il permis d'espérer? La première question n'est bien sûr que spéculative (bloß spekulativ). Kant se flatte d'avoir épuisé par sa Critique toutes les réponses possibles à cette question, mais alors, ajoute-t-il, «nous sommes restés tout aussi éloignés des deux grandes fins où tendait proprement tout cet effort de la raison pure» ${ }^{32}$ (les deux grandes fins dont s'enquiert la double question, «y a-t-il un Dieu? y a-t-il une vie future?»). Kant règle ensuite un peu rapidement le cas de la seconde question où s'exprime l'intérêt de la raison: que dois-je faire? Reprenant les termes employés pour diminuer l'urgence de la première question, il écrit: «la seconde question n'est que pratique (bloß praktisch)». Comme telle, déclaret-il, elle n'appartient pas à la raison pure. Ce n'est donc pas une question transcendantale, mais morale, qui n'a pas à occuper notre critique. La seconde question se trouve ainsi provisoirement écartée de la discussion du «fondement pour la détermination de la fin dernière de la raison».

Toute l'attention de Kant se portera sur la troisième question: «si je fais ce que je dois, que m'est-il alors permis d'espérer?» Cette troisième question offre l'avantage d'être à la fois pratique et théorique. Pratique, car l'espoir investit toujours ce que nous voulons et devons faire, mais théorique aussi, puisque l'objet visé par l'espérance doit relever d'une quelconque connaissance, qu'il s'agira maintenant de délimiter. Ce qui intéresse manifestement Kant, c'est que le pratique serve un peu de relais au théorique, «de telle sorte que le pratique ne conduit que comme un fil conducteur à la solution de la question théorique» ${ }^{33}$. Kant envisage donc, à ne pas s'y tromper, une solution à la question théorique par le biais de l'usage pratique de la raison pure. On trouve, en filigrane, dans ce relais le noeud de la solution kantienne au problème de la métaphysique. Il est déjà révélateur que toute la seconde section du Canon n'ait trait qu'à la troisième question, celle de l'espérance. C'est elle qui renferme la clef du problème initial de Kant.

Que vise donc l'espoir? Kant répond, dans les termes les plus simples: «tout espoir tend au bonheur» ${ }^{34}$. Réaliste ou pessimiste, Kant estime cependant que notre quête du

33 Ibid.

34 Ibid. 
bonheur ne sera jamais pleinement assouvie sous des conditions empiriques (et qui voudrait dire qu'il a tort?). Le bonheur suprême que chacun désire n'en est pas un qui pourrait être satisfait en notre existence terrestre. Il est possible de traduire littéralement le mot Glückseligkeit qu'emploie Kant par «bonheur de félicité». Ce qui est espéré par notre raison, ce n'est pas une plus grande jouissance ou un simple bien-être matériels, mais une tranquillité d'âme qui n'est pas que de ce monde. Pour sûr, nous ne pouvons pas être nous-mêmes les artisans d'un tel bonheur, qu'on pourrait qualifier de souverain. Tout ce que nous pouvons faire, et espérer, c'est de nous rendre dignes d'un tel bonheur.

Kant répond par le fait même à la question «que dois-je faire?» qu'il avait professé d'écarter de la philosophie transcendantale: «fais ce qui peut te rendre digne d'être heureux» ${ }^{35}$. Et agir afin de se rendre digne du bonheur, c'est se conformer, autant que possible, à l'esprit de la loi morale produite par la raison pure pratique. L'ultime objet de notre espérance consistera donc pour la raison pure dans l'attribution d'un bonheur éternel proportionnellement lié à la moralité de nos actions. Pareil espoir n'a de sens que si on admet l'existence d'une raison suprême en mesure d'assurer le «lien nécessaire ... de l'espoir d'être heureux avec l'effort incessant pour se rendre digne du bonheur» ${ }^{36}$. C'est ce lien, entre l'espoir de la raison et sa réalisation, qu'incarne l'idéal du bonheur souverain dans la première Critique: «l'idée d'une telle intelligence où la volonté moralement la plus parfaite, liée à la suprême félicité, est la cause de tout bonheur dans le monde, en tant que ce bonheur est exactement proportionné à la moralité (comme à ce qui rend digne d'être heureux), je l'appelle l'idéal du souverain bien» ${ }^{37}$. On retrouve ici la doctrine bien connue des postulats de la raison pratique qui couronnera la seconde Critique. On découvre cependant que l'essentiel de cette doctrine se trouve déjà dans la Critique de 1781, dont elle constitue la véritable conclusion.

Seulement, l'optique de 1781 dévie de celle de 1788 sur un point important. Tandis que la seconde Critique fera du respect de la loi morale pour elle-même, à l'exclusion de toute récompense future, le seul motif légitime de l'action morale, le Canon de 1781 voit encore dans le souverain bien et sa promesse d'une félicité future des mobiles nécessaires à l'action morale. Beaucoup moins rigoriste que la Critique de la raison pratique, la méthodologie de 1781 est ainsi plus proche de la morale classique: «Sans un Dieu et sans un monde qui n'est pas maintenant visible pour nous, mais que nous espérons, les magnifiques idées de la moralité peuvent donc bien être des objets d'approbation et d'admiration, mais ce ne sont pas des mobiles (Triebfedern) de l'intention et de l'exécution, parce qu'elles n'accomplissent pas la fin dernière qui est assignée a priori précisément par cette même raison pure et qui est nécessaire» ${ }^{38}$. Même si l'idée d'une morale autonome qui n'a pas besoin de l'espoir d'une gratification future commence à

35 A 809/B 837; Euvres, 1368; TP 545.

${ }_{36}$ A 810/B 838; Euvres, 1369; TP 546.

37 Ibid.

38 A 813/B 841; Euvres, 1371; TP 547. 
s'y faire jour ${ }^{39}$ - signe d'une tension non résorbée dans l'œuvre de 1781 et peut-être dans tout le kantisme -, la première Critique se réclame expressis verbis d'un «système de la moralité qui se récompense elle-même» ${ }^{40}$, estimant que l'hypothèse d'un sage gouverneur est une «nécessité pratique» afin «de donner [aux lois morales] leur effet» ${ }^{41}$. La raison, écrit encore Kant, se voit forcée «ou d'admettre un tel être, ainsi que la vie dans un monde que nous devons considérer comme futur, ou de regarder les lois morales comme de vaines chimères ${ }^{42}$. Chacun doit donc considérer les lois morales comme des commandements qui comportent «des promesses et des menaces» ${ }^{43}$.

C'est ainsi que le souverain bien, pensé dans la perspective de 1781, permet d'envisager une réponse positive à la double question de la raison pure: y a-t-il un Dieu? y a-t-il une vie future? On comprend aussi le sens du titre de la deuxième section du Canon, la clef de voûte de la métaphysique kantienne: «De l'idéal du souverain bien comme principe qui détermine la fin suprême de la raison». L'espoir qu'aménage le principe du souverain bien permet, en effet, de fonder sur le sol de la raison pure pratique ce que Kant appelle avec insistance les «deux propositions cardinales de notre raison pure: il y a un Dieu, il y a une vie future ${ }^{44}$. C'est, dans la Critique de la raison pure, la réponse la plus claire qui soit apportée au problème fondamental de la métaphysique.

Mais, pourrait-on se demander, ces deux propositions cardinales ont-elles été véritablement fondées? Ces deux affirmations, que n'a pas pu établir la raison seulement théorique, sont de toute évidence des propositions synthétiques a priori. Or c'était pour jauger la possibilité de tels jugements qu'une critique transcendantale avait été mise en place. Comment justifier de telles assertions métaphysiques devant le tribunal d'une critique de la raison pure? Nous savons qu'un troisième terme est nécessaire en vue d'expliquer le bien-fondé d'une proposition synthétique a priori. Quel peut bien être ce tertium quid dans le cas des postulats de la raison pratique? La première Critique n'est pas très diserte sur cette question, tout n'y étant encore que programme. On peut donc légitimement s'inspirer de la réponse qu'offrira la seconde Critique à notre question: «Pour étendre pratiquement une connaissance pure, il faut que soit donnée a priori une intention (Absicht), c'est-à-dire un but comme objet (de la volonté) qui, indépendamment de tous les principes théoriques, soit représenté comme pratiquement nécessaire par un impératif déterminant la volonté immédiatement (catégorique); et c'est ici le

39 En A 813-4 / B 841-2; CEuvres, 1371; TP 548: «c'est la disposition morale qui rend possible, comme condition, la participation au bonheur, et non pas, à l'inverse, la perspective du bonheur qui rend d'abord possible la disposition morale». Ce texte nous paraît difficilement conciliable avec celui que nous venons de citer (note 38) et ceux que nous citerons dans la fin de ce paragraphe (notes 40 à 43).

40 A 809/B 837; Cuvres, 1368; TP 546.

41 A 818/B 846; CEuvres, 1375; TP 550-1.

42 A 811 / B 839; Euvres, 1370; TP 547.

${ }^{43}$ Ibid.

${ }_{44}$ A 741 / B 769; Euvres, 1319; TP 509. Cf. aussi A 811 / B 839; Euvres, 1369; TP 546 et A 830 / B 858; Euvres, 1383; TP 557. 
souverain bien ${ }^{45}$. Ce qui autorise l'extension a priori du savoir dans un horizon pratique, c'est donc une $A b s i c h t$, un intérêt a priori de la raison, la double fin: y a-t-il un Dieu? y a-t-il une vie future? Non seulement l'objet de la métaphysique nouvelle est-il conçu en fonction des intérêts de la raison, la possibilité même de la métaphysique et du synthétique a priori découlant de l'ordre pratique sera explicitement fondée sur l'intention suprême de l'humanité, le souverain bien. Le summum bonum de la Méthodologie et de la seconde Critique incarne aussi bien le but que la condition de possibilité de la métaphysique.

Mais est-il légitime de conclure d'un besoin à la réalité de son objet? L'homme est-il immortel du seul fait qu'il le désire? L'existence de Dieu est-elle prouvée parce qu'elle apparaît nécessaire à la réalisation du souverain bien conçu par l'homme? L'auteur qui a dénoncé tout au long de la Dialectique l'illusion transcendantale qui consiste à prendre ses désirs métaphysiques pour des réalités succombe-t-il au même sophisme? À n'en pas douter, la métaphysique des postulats de la raison pratique procède d'un intérêt de la raison. Kant serait le dernier à le contester. Mais comment justifier le saut de l'intention à la réalité d'un objet qui ne peut être donné en aucune expérience?

La Méthodologie de 1781 n'évoque pas encore cette objection, assurément la plus sérieuse qui puisse être soulevée en regard d'une philosophie qui se met de propos délibéré sous l'égide de l'intérêt métaphysique de la raison. Mais Kant s'y attaquera dans une note de la deuxième Critique où il renvoie à une étude du philosophe Winzenmann qui «conteste le droit de conclure d'un besoin à la réalité objective de l'objet, et explique sa pensée par l'exemple d'un amoureux qui, s'étant épris à la folie d'une idée de beauté qui n'est qu'une chimère de son cerveau, voudrait conclure qu'un objet de ce genre existe réellement quelque part» ${ }^{46}$. La critique de Winzenmann s'en prend évidemment à la métaphysique pratique élaborée dans le Canon de la première Critique, sept ans avant la Critique de la raison pratique où Kant répond déjà aux critiques de la théologie morale esquissée en 1781. Kant se dit pleinement d'accord avec Winzenmann pour ce qui est des désirs strictement empiriques et contingents. «Je lui donne parfaitement raison dans tous les cas où le besoin est fondé sur l'inclination, qui ne peut même pas postuler nécessairement, du point du vue de celui qui en est affecté, l'existence de son objet et contient encore moins une exigence valable pour chacun et qui, par conséquent, n'est qu'un principe subjectif des souhaits» ${ }^{47}$. De l'inclination à la réalité, la conséquence n'est pas bonne. Mais l'intérêt qui nous pousse à postuler l'existence de Dieu et l'immortalité de l'âme n'est pas un penchant comme les autres. Il n'est pas ici question, nous assure Kant, d'un penchant subjectif, donc empirique et particulier, mais d'un Vernunftbedürfnis, d'un «besoin rationnel dérivant d'un principe déterminant objectif de la volonté, c'est-à-dire de la loi morale, laquelle oblige nécessairement tout être raisonnable, et, par conséquent, l'autorise à supposer a priori dans la

45 Kritik der praktischen Vernunft, Ak V, 134; trad. (modifiée ici) par L. Ferry et H. Wismann, en E. Kant, Euvres philosophiques, t. II, Gallimard, Bibliothèque de la Pléiade, Paris, 1985, 771.

${ }^{46}$ Ak V, 143-4; CEuvres, t. II, 783.

47 Ibid. 
nature des conditions conformes à la loi, et rend ces dernières inséparables de l'usage pratique complet de la raison ${ }^{48}$. Autrement mis, l'intérêt de la raison est objectif parce qu'universel, faisant système avec le seul a priori positif de la raison pure, la loi morale. Le commandement moral, factum de la raison, a pour telos ultime le souverain bien. Laisser tomber l'objectif universel de la loi morale équivaudrait, dans l'esprit de Kant, à priver la raison de tout efficace et, finalement, de toute cohérence. Pourquoi la raison enjoindrait-elle tous les êtres moraux à travailler à la réalisation d'un idéal impossible? Acquiescer à la loi morale, principe de l'action, c'est en même temps admettre son terme, le souverain bien et sa double condition de possibilité, Dieu et l'immortalité, leur supposition étant «aussi nécessaire que la loi morale» ${ }^{49}$. L'objectivité des postulats de la raison pratique dérive de l'indubitable objectivité, c'est-à-dire ici de l'universalité, de la loi morale et de la rationalité qu'elle ouvre à l'existence humaine. De l'espérance à la réalité objective de ce qui est espéré, la conclusion n'est bonne que s'il s'agit d'un besoin objectif de la raison et Kant n'en connaît qu'un seul, le bien suprême, lié à deux articles de foi, l'existence de Dieu et l'immortalité de l'âme. On trouve dans cette supposition, dans cette logique de la rationalité pratique, la conclusion d'une critique de la raison pure, dont l'intention était d'évaluer l'usage légitime et l'extension possible de la raison pure. C'est le point d'aboutissement, limité, mais inestimable, de la première Critique, qui répond ainsi à la question centrale de la possibilité de la métaphysique pour la raison humaine.

Mais cette issue de la métaphysique kantienne ne saurait en aucune façon donner lieu à un triomphalisme de la raison pure. Le postulat pratique de l'existence de Dieu, non sa pénétration théorique, et de l'immortalité résume tout ce que peut espérer la raison lorsqu'elle élabore l'idéal du souverain bien. Paraphrasant un passage des Paralogismes, on pourrait affirmer que la proposition «il y a un Dieu, il y a une vie future» constitue «le seul texte» de la théologie pratico-rationnelle.

Cette modeste conclusion ne paraîtra étroite qu'aux yeux des écoles rationalistes, beaucoup plus ambitieuses dans leurs recherches a priori, mais Kant estime qu'elle suffit parfaitement si l'on veut répondre - et la philosophie n'a pas d'autre tâche - aux intérêts de la raison et à ce que le commun des mortels attend de la métaphysique. Ce sera la conclusion du Canon de la raison pure et, partant, de la première Critique: «Mais est-ce là, dira-t-on, tout ce qu'accomplit la raison pure, quand elle s'ouvre des vues par-delà les limites de l'expérience? Rien que deux articles de foi? L'entendement commun en aurait bien pu faire autant, sans avoir besoin de consulter là-dessus les philosophes! ... Mais exigez-vous donc qu'une connaissance qui regarde tous les hommes surpasse l'entendement commun et ne puisse vous être révélée que par les philosophes?» ${ }^{50}$. La métaphysique des intérêts de la raison ne prétend pas transcender le sens commun, elle

${ }^{48}$ Kritik der praktischen Vernunft, Ak V, 144; Cuvres, t. II, 783. On se reportera aussi à Qu'est-ce que s'orienter dans la pensée?, où Kant défendra «le droit du besoin de la raison» (Ak VIII, 137; Euvres, t. II, 534) à orienter l'extension de la pensée en dehors des limites de l'expérience.

${ }^{49}$ Kritik der praktischen Vernunft, Ak V, 144; Euvres, t. II, 783.

50 A 830-1 / B 858-9; Euvres, 1383-4; TP 557. 
se met plutôt à son service pour en être le défenseur et le porte-parole. C'est la conséquence, et la nouveauté peut-être, de l'inflexion pratique de la métaphysique et de la redéfinition de la philosophie dans un sens non plus scolaire, mais «cosmique», comme «science du rapport de toute connaissance aux fins essentielles de la raison humaine (teleologia rationis bumanae) $\aleph^{51}$. Et «la plus haute philosophie, par rapport aux fins essentielles de la nature humaine, ne peut pas conduire plus loin que ne le fait la direction qu'elle a remise à l'entendement commun" ${ }^{52}$.

La critique transcendantale de Kant aboutit ainsi à une réorientation de la philosophie et à une accentuation pratique de la métaphysique. Les postulats métaphysiques de l'existence de Dieu et de l'immortalité ne sont légitimes que parce qu'ils s'inscrivent dans la logique du seul a priori positif de la raison, la loi morale. Seul l'a priori moral rend praticable un dépassement de l'expérience et l'hypothèse de deux principes transcendants. Mais ce résultat de la réflexion kantienne sur l'a priori pratique de notre raison est-il compatible avec la leçon de la Logique transcendantale, qui limitait sans équivoque le champ du savoir a priori à celui de l'expérience possible, selon le texte de B 166 que Kant n'avait pas manqué de souligner: «il n'y a de connaissance a priori possible pour nous que celle d'objets d'expérience possible»? Cette thèse dit bien qu'aucun savoir a priori ne peut outrepasser l'horizon de l'expérience possible. C'est se tirer d'affaire à trop bon compte que de déclamer que les postulats relèvent du «pratique» et non du théorique, car les propositions «il y a un Dieu, il y a une vie future» nous livrent bien un enseignement, une «doctrine», qui est nécessairement d'ordre théorique (la preuve c'est qu'une métaphysique en sera possible), même si sa pierre de touche réside bel et bien dans un commandement pratique. Comment concilier le «résultat» de la Logique et celui de la Méthodologie, qui prête une réalité objective aux postulats métaphysiques, donc a priori, de l'immortalité et de l'existence de Dieu? Les dernières pages de la Critique ont-elles choisi d'ignorer la leçon de l'Analytique, logique de la vérité?

Il nous semble que non et que Kant était plutôt tout à fait conscient de la compatibilité de sa logique de la vérité et de sa Méthodologie. C'est pour souligner la cohérence de son entreprise critique qu'il prend la peine d'écrire, presque stratégiquement, dans le Canon de la raison pure - en conformité avec le libellé de B 166 - que les principes tirés de la raison pratique sont «des principes de la possibilité de l'expérience» ${ }^{53}$. À l'instar de la métaphysique théorique de la nature, celle de l'Analytique, la métaphysique pratique a pour fonction d'épeler les conditions de l'expérience possible, expérience qui n'est pas uniquement celle de la science physique newtonienne, comme on le proclame souvent, mais qui est aussi, et primordialement, celle de la praxis. Et pour Kant, cette «expérience pratique» est celle de la liberté. Présentée parfois comme la troisième grande idée de la raison avec Dieu et l'immortalité, la liberté a l'avantage, dit

51 A 839/B 867; Euvres, 1389; TP 562.

52 A 831 / B 859; CEuvres, 1384; TP 557.

53 A 807/B 835; CEuvres, 1367; TP 544. Le souligné est de Kant. 
Kant, d'être la seule des idées qui soit directement démontrée par l'expérience ${ }^{54}$. La liberté pratique avait été définie, dans l'Antinomie, comme l'indépendance de la volonté par rapport à la contrainte des pulsions de la sensibilité ${ }^{55}$. Le simple fait qu'il nous arrive de délibérer de ce qui est désirable, bon ou raisonnable, suffit pour établir l'existence d'une liberté pratique. L' «expérience possible» qui intéresse la métaphysique du pratique est donc celle de la liberté (d'où l'idée d'un «système de la liberté» ${ }^{56}$ dans le Canon). La méditation sur les a priori de la liberté n'aura qu'à montrer que la loi morale en incarne la ratio cognoscendi et le souverain bien le telos ultime. Les postulats du souverain bien ne sont par conséquent que des conditions de l'exercice de la liberté.

Ils n'en représentent pas moins l'issue métaphysique, la conclusion théiste de la Critique de la raison pure. Au reste, Kant présentera souvent cet aboutissement théologique de la rationalité pratique comme la clef de voûte de la philosophie transcendantale. Retraçant en 1793 l'évolution de la métaphysique en Allemagne, qui culmine dans sa propre philosophie, Kant soutient que le troisième et dernier stade de la métaphysique «est celui de la théologie, avec toutes les connaissance a priori qui y conduisent et la rendent nécessaire», celui «où la métaphysique donne à son trajet un accomplissement pratiquement dogmatique et où elle parvient à sa fin ultime» ${ }^{57}$. C'est sur le théologique que veut s'achever la philosophie critique, souligne déjà Kant dans le dernier chapitre de la Critique, roulant sur l'Histoire de la raison pure: «Il est assez remarquable, bien que cela ne pût arriver naturellement d'une autre manière, que les hommes, dans l'enfance de la philosophie, aient commencé par où nous finirions plutôt maintenant, c'est-à-dire par étudier la connaissance de Dieu et l'espérance» ${ }^{58}$. N'est-il pas significatif que les trois Critiques se terminent, à toutes fins utiles, sur des «preuves» de l'existence de Dieu: en 1781, dans le Canon de la raison pure et plus spécifiquement dans l'idéal du souverain bien comme principe qui détermine «la fin dernière de la raison pure», en 1788, dans la doctrine des postulats de la raison pratique et dans la Critique du jugement sur une apologie de la théologie morale et, selon l'intitulé de son dernier paragraphe, sur une étude de «l'assentiment résultant d'une croyance pratique»? Est-ce un hasard si le premier livre majeur que Kant fait paraître après avoir clos son œuvre critique en 1790 , alors que tous attendaient la métaphysique promise en 1781 , traite de la «religion dans les limites de la simple raison»? La préoccupation théologique, l'héritage le plus intime de la métaphysique classique, a aussi surplombé les tout derniers travaux du penseur, ceux de l'Opus postumum. Les titres que Kant envisageait de donner à son dernier ouvrage, qui promettait d'être une présentation systématique de la philosophie transcendantale, confirment l'orientation ultimement théologique de sa spéculation: «La totalité des choses: Dieu et le monde», «Dieu, le monde et

${ }_{54}$ A 802/B 830; CEuvres, 1363; TP 541.

55 A 534/B 562; Euvres, 1168; TP 395.

56 A 815/B 843; CEuvres, 1373; TP 549.

57 Progrès de la métaphysique, Ak XX, 7, 281; trad. par J.Rivelaygue, in E. Kant, CEuvres philosophiques, t. III, Gallimard, Bibliothèque de la Pléiade, Paris, 1986, 1235.

58 A 852 / B 880; Euvres, 1399; TP 569. C'est nous qui soulignons. 
l'homme», «Système total de la raison pure» ${ }^{59}$. Le doublet «Dieu et le monde» est le leitmotiv qui revient tout au long de l'Opus postumum: «la philosophie transcendantale: la doctrine de Dieu et du monde» ${ }^{60}$. H.J. De Vleeschauwer a donc raison de soutenir que l'idée de Dieu devait incarner le sommet de l'Opus ${ }^{61}$. L'idéal théologique, dernier moment de la raison pure dans la Dialectique, devait aussi couronner le système de la philosophie transcendantale, solidaire en cela de l'impulsion fondamentale de la métaphysique et dont la possibilité devait être établie par la propédeutique d'une critique de la raison pure. C'est la métaphysique que voulait rendre possible le Canon de la raison pure, la conclusion de la première Critique.

$*$

Le but de notre étude n'a pas été d'«inventer» une conclusion à un ouvrage qui n'en comporte aucune. Le problème de la «conclusion» de la Critique de la raison pure, que nous avons voulu soulever ici, n'en est pas un de composition littéraire, mais de fond: il s'agit de savoir en quoi consiste la réponse de Kant à la question de la possibilité de la métaphysique comme science respectable. La métaphysique à laquelle on a affaire chez Kant doit, si elle est possible, pouvoir offrir une réponse aux questions les plus essentielles, les plus intéressées de la raison pure: y a-t-il un Dieu? y a-t-il une vie future? Si Kant propose une critique de la raison pure, c'est pour élucider le fondement possible d'une science qui sache répondre à ces interrogations de la metaphysica naturalis. Au moment d'écrire la première Critique, Kant ignore qu'il publiera encore deux autres Critiques. Dans la perspective de 1781, une seule Critique, qui veut épuiser toutes les possibilités de la raison pure, doit suffire à l'essor de la métaphysique. En tant que traité de la méthode, la Critique a bel et bien enseigné une nouvelle voie à la métaphysique future. Dès la Critique de 1781, cette voie est celle de la raison pure pratique, efficace dans son commandement impératif et dans la cohérence du système de la moralité que parachève l'idéal du souverain bien. Depuis ce «principe» du souverain bien, Kant tentera de répondre aux problèmes premiers de la métaphysique. C'est donc le Canon qui résout la question de la possibilité de la métaphysique. Ceci trouve aussi confirmation dans le caractère presque dramatique de sa diction ( $« s^{\prime}$ il y a quelque part un usage légitime de la raison pure ... il doit y avoir aussi un canon ...» et ses titres «De la fin dernière de l'usage pur de notre raison», «De l'idéal du souverain bien comme fondement pour la détermination de la fin dernière de la raison pure», etc.). Après ce Canon pourront apparaître une Architectonique et une Histoire de la raison pure qui présenteront de manière parfaitement schématique le plan du système de la raison que vient de rendre possible le Canon. À la limite, on pourrait considérer ces derniers chapitres comme des «appendices» à la Critique de la raison pure et à sa conclusion, le Canon de la raison pure.

59 Pour les références, multiples, cf. H.J. De Vleeschauwer, La déduction transcendantale dans l'cuvre de Kant, t. III, Librairie Ernest Leroux, Paris, 1937, 662.

60 Opus postumum, Ak XXI, 6 et passim.

${ }^{61}$ H.J. De Vleeschauwer, La déduction transcendantale, III, 651. 